\title{
Risk and rewards of fecal transplants
}

$\mathrm{T}$ he "yuck" factor alone compels many to instinctively want to wash their hands, and/or wash their hands of it.

A Calgary, Alberta, doctor performs the procedure in people's homes, to avoid administrative squabbles with local health officials. A Burnaby, British Columbia hospitalist is now embroiled in just such a dust-up with her local health authority over whether the procedure is too unproven and risky to be offered to patients.

Meanwhile, a Toronto, Ontario, epidemiologist is currently recruiting patients for the first North American randomized, controlled trial on the procedure.

It's called fecal bacteriotherapy and more commonly known as a fecal transplant. It involves the introduction of saline-diluted fecal matter from a donor into a patient's gastrointestinal tract via a nasoduodenal catheter or enema, primarily to treat Clostridium difficile infection.

For the last few decades, a small number of doctors have used the treatment as salvage therapy in North America. The treatment is slightly more popular in Scandinavia and published cases have emerged from approximately 20 sites around the world. They suggest fecal transplants can be life-saving for patients with recurrent $C$. difficile.

Dr. Thomas Louie, head of infectious disease at Calgary's Foothills Hospital, performed his first fecal transplant in 1996 at the home of a patient who'd been suffering from a $C$. difficile infection for two years, after an administrator informed him that it couldn't be done in the hospital. He's since performed 70 such home treatments, mostly by enema, on a volunteer basis, nights and weekends. "I'm taking the risk myself," he says.

Louie screens donors for infectious diseases and their stool for ova and parasites. Donors must be a relative because Louie noticed that patients tend to have a longer post-transplant recovery period if the donor isn't genetically related. Louie now collects samples of donor and recipient

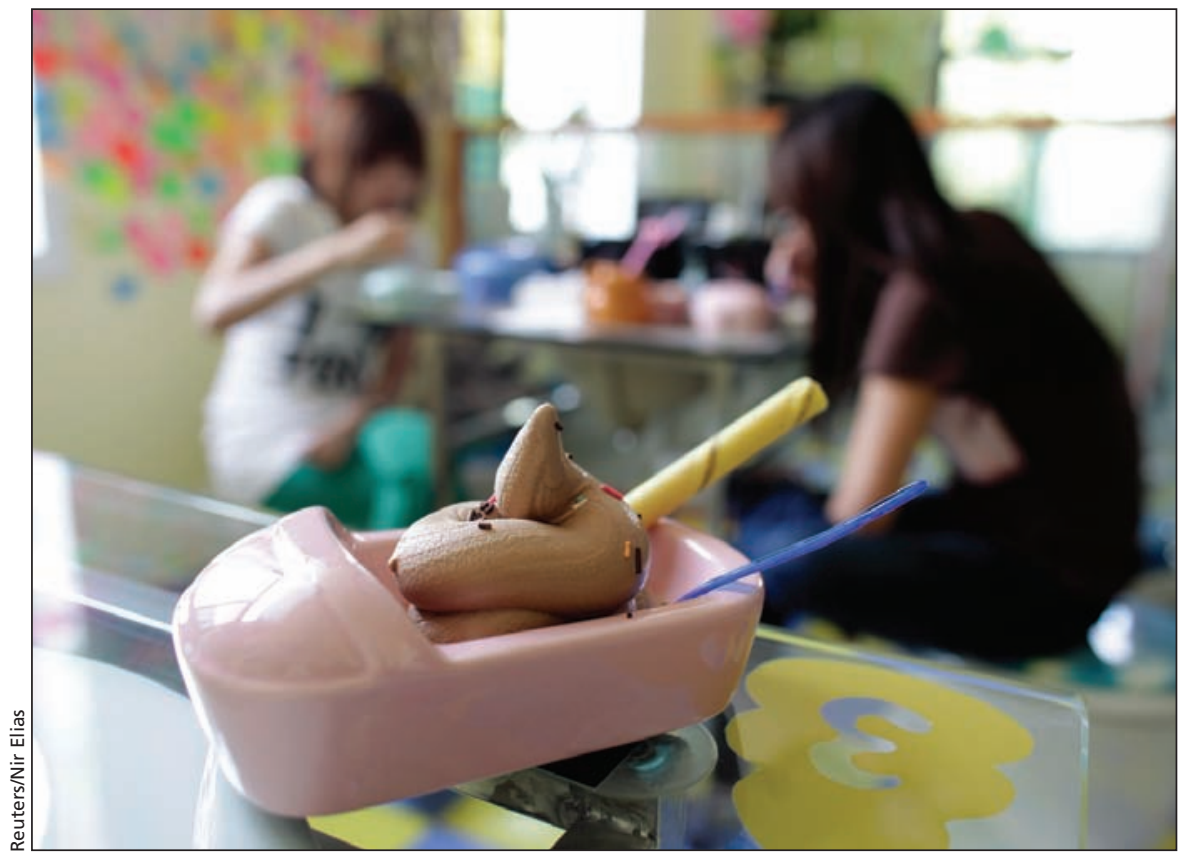

An ice cream shaped as feces is displayed at a toilet-themed restaurant in Beijing, China During fecal transplants, fecal matter is typically transferred from a volunteer donor into the colon of the infected patient but it can also be administered by the mouth or the nose.

stools before and after the procedure as part of a study to determine "which of the bugs are accepted and which are rejected."

Meanwhile Burnaby's Dr. Jeanne Keegan-Henry performed a fecal transplant in 2010 without getting approval from the local health authority, Fraser Health. Keegan-Henry claims the procedure cured the patient of recurrent $C$. difficile and would now like to offer the treatment more broadly, on compassionate grounds. But her local health authority forbade her from performing another treatment without an approved research proposal. In Canada, hospitals and local health authorities decide whether to take on the risk associated with an experimental treatment.

"At any given time, there are always some people sick in the hospital with C. difficile," says Keegan-Henry, noting that $40 \%$ of patients fail first-line treatment and $40 \%$ of remaining patients fail second-line treatment. "Patients have died because they didn't get access to this procedure."

But Dr. Andrew Webb, vice president of medicine at Fraser Health, says the evidence isn't strong enough to sup- port fecal transplants as a rescue therapy. What literature exists comes from nonrandomized case studies, he says. "With these studies, you tend to report the positive and not the negatives. ... There's a rather long list of nasty infections that can be transmitted from bodily fluids."

Dr. Susy Hota, epidemiologist at Toronto General Hospital, hopes that her proposed randomized control trial on fecal bacteriotherapy, and one underway in the Netherlands, will make Canadian doctors more comfortable with the treatment and standardize the procedure.

The trial, which is seeking 146 patients who suffer from recurrent $C$. difficile infection and who have failed at least 10 days of vancomycin treatment, will screen donor blood for such infectious diseases as HIV I and II, hepatitis $\mathrm{A}, \mathrm{B}$, and $\mathrm{C}$ and donor stool for diarrhea-causing organisms such as salmonella, shigella and parasites.

Hota says a randomized controlled trial hasn't been conducted in North America to date because $C$. difficile outbreaks tend to be sporadic, small and isolated, and obtaining funding for 
such a trial is problematic because it doesn't involve a drug that pharma can profit from.

Then there's the yuck factor, she adds. "No hospital would support doing this study on their grounds except for ours."

C. difficile is a bacteria that is believed to cause diarrhea and such intestinal diseases as colitis when normal bacteria in the gut are eradicated by antibiotics. $C$. difficile spores may be ingested by patients in hospitals, nursing homes and health care settings. Severe infections are typically treated by vancomycin or metronidazole.

But Hota says the etiology of $C$. difficile infections is unclear and the study hopes to pinpoint the causes. "We think it's more than just the bacteria."

A review of 100 Scandinavian cases found that fecal bacteriotherapy cured
89\% of patients (Anaerobe. 2009;15: 285-90). Dr. Johan Bakken, the review author and a gastroenterologist in Duluth, Minnesota, says the risk of transmitting a contagious agent through fecal bacteriotherapy is merely "theoretical."

Bakken says there hasn't been a single reported case of a transmitted infection agent. He also estimates that more than 500 unpublished fecal transplants have occurred, most of those without donor screening because of its high cost. In most sites where a fecal transplants are undertaken, donors are a "bed or table contact" of the infected individual, to minimize the risk of disease transmission, Bakken adds.

Not so at St. Joseph's Hospital in Hamilton, Ontario, where doctors have relied on a small pool of screened donors for material used to undertake more than 50 fecal transplants over the past 18 months. "Organizing and mobilizing families for transplants was challenging," says Dr. David Higgins, the hospital's chief of staff. "It felt unfair to offer it to some people and not others."

Higgins says $90 \%$ of the patients treated have been effectively cured and there hasn't been any known transmission of infectious diseases. "We're getting institutions contacting us from across the country to see if we can deal with other patients."

As for the yuck factor, Bakken notes that patients aren't squeamish. "Those that voice objection to the therapy are not the patients themselves because they are at last straw." - Wendy Glauser, Toronto, Ont.

CMAJ 2011. DOI:10.1503/cmaj.109-3806

\section{Patient safety database goes online}

A Canadian-developed, Webbased patient safety alert database has been launched to collate information about harmful incidents from around the world in hopes it will spur reforms to prevent similar incidents in the future.

The publicly accessible site, Global Patient Safety Alerts, will contain summaries and links to more than 900 recent patient safety advisories, alerts and recommendations on incidents in which a patient was harmed, or had the potential to be harmed, from some 22 international, national and regional health authorities and organizations worldwide (www.globalpatientsafety alerts.com).

The Canadian Patient Safety Institute (CPSI) developed the site to give health care providers and policy-makers an opportunity to become aware of adverse events, identify similarities and patterns in sources of risk, share solutions and ultimately, prevent similar ones from occurring in the future.

"It's transparency gone wild," says institute CEO Hugh MacLeod. "The reality is we're working in very com-

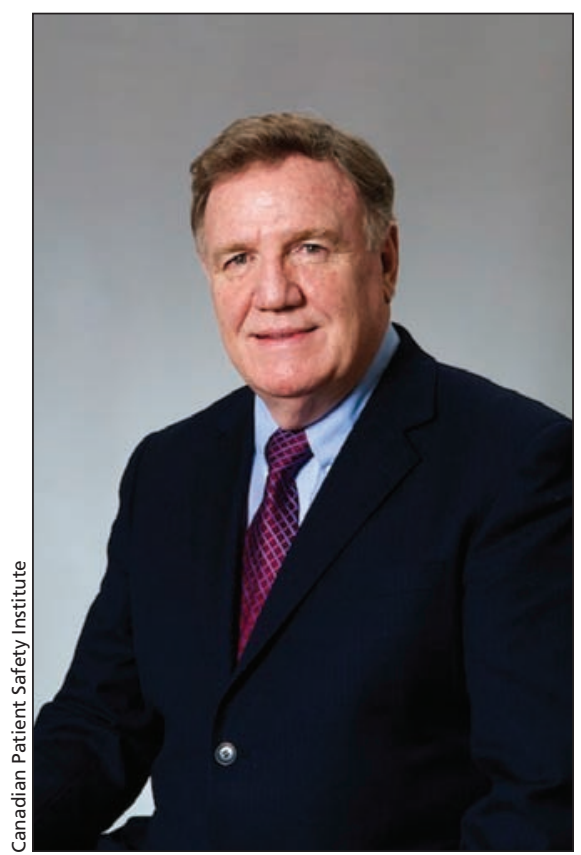

"It's transparency gone wild," says Canadian Patient Safety Institute CEO Hugh MacLeod.

plex, fast-moving systems. We have new drugs, new technologies, but also new demands and expectations from the public, and mistakes happen. Now, however, we can begin to share, ana- lyze and learn from those mistakes on a broader scale."

Historically, when an incident occurred in one jurisdiction, other regions were not made aware of the mistake, and did not benefit from the lessons learned, because no global system existed to gather and share the information, says Paula Beard, CPSI director of operations, citing recent oxygen tube mix-ups as an example. "Incidents were happening on a oneoff basis in hospitals across Canada. What we've now found out through our system is that they were also happening around the world and a number of solutions had been implemented, but there wasn't any coordination between the organizations that were trying to solve that problem."

The website aggregates data from groups that produce $80 \%$ to $85 \%$ of global patient safety information, including the health authorities and incident reporting systems of the European Union, United Kingdom, Australia and a number of American states. It also collates patient safety alerts from organizations such as the 